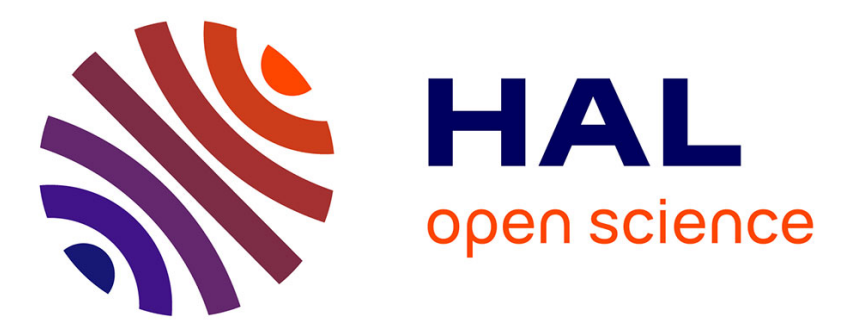

\title{
On-Line Hybrid Cramer-Rao Bound for Oversampled Dynamical Phase and Frequency Offset Estimation
}

\author{
Jordi Vilà Valls, Jean-Marc Brossier, Laurent Ros
}

\section{To cite this version:}

Jordi Vilà Valls, Jean-Marc Brossier, Laurent Ros. On-Line Hybrid Cramer-Rao Bound for Oversampled Dynamical Phase and Frequency Offset Estimation. Globecom 2009 - IEEE Global Communication Conference (Globecom'09), Nov 2009, Honolulu, Hawaii, United States. pp.1-6, 10.1109/GLOCOM.2009.5425731 . hal-00447402

\section{HAL Id: hal-00447402 \\ https://hal.science/hal-00447402}

Submitted on 14 Jan 2010

HAL is a multi-disciplinary open access archive for the deposit and dissemination of scientific research documents, whether they are published or not. The documents may come from teaching and research institutions in France or abroad, or from public or private research centers.
L'archive ouverte pluridisciplinaire HAL, est destinée au dépôt et à la diffusion de documents scientifiques de niveau recherche, publiés ou non, émanant des établissements d'enseignement et de recherche français ou étrangers, des laboratoires publics ou privés. 


\title{
On-line Hybrid Cramér-Rao bound for oversampled dynamical phase and frequency offset estimation
}

\author{
Jordi Vilà Valls, Jean-Marc Brossier and Laurent Ros \\ GIPSA-lab, Departement Image Signal \\ BP 46 - 38402 Saint Martin d'Hères - FRANCE \\ E-mail: jordi.vilavalls@gipsa-lab.inpg.fr, jean-marc.brossier@gipsa-lab.inpg.fr, laurent.ros@gipsa-lab.inpg.fr
}

\begin{abstract}
This paper deals with the on-line estimation of a dynamical carrier phase and a frequency offset in a digital receiver. We consider a Brownian phase evolution with a linear drift in a Data Aided scenario. The proposed study is relative to the use of an oversampled signal model after matched filtering, leading to a coloured reception noise and a non-stationary power signal. We derive a closed-form expression of the Hybrid CramérRao Bound (HCRB) for this estimation problem. We use a Binary Offset Carrier (BOC) function as shaping pulse. Our numerical results show the potential gain of using the oversampled signal for estimating the dynamical phase and frequency offset, obtaining better performances than using a classical synchronizer.

Index Terms-Phase estimation, HCRB, oversampling, carrier synchronization, GALILEO, BOC
\end{abstract}

\section{INTRODUCTION}

Synchronization is a fundamental part in modern digital receivers. A synchronizer has to estimate some parameters such as carrier frequency, carrier phase and timing epoch. This knowledge is required to recover the signal of interest correctly. In this paper we focus our attention on the dynamical phase and frequency offset estimation problem. When having an estimation process we need lower bounds on the estimation performance to be used as a benchmark. The family of Cramér-Rao Bounds (CRBs) has been shown to give accurate estimation lower bounds in many scenarios [1].

Several Cramér-Rao lower bounds have appeared in the literature.

For constant phase offset estimation in the so-called DataAided (DA) scenario, Rife et al. [9] derive CRB closedform expressions; Cowley [10] does so in the Non-Data-Aided (NDA) scenario.

For time-varying parameter estimation, an analytical expression of a general on-line recursive Bayesian CRB (BCRB) is given by Tichavský [8]. Bay et al. [6] introduced an Asymptotic BCRB(ABCRB), provide an analytical expression of the off-line $\mathrm{CRB}$ and $\mathrm{BCRB}$, and derived the general formulation for the Hybrid CRB (HCRB) [7], useful when having both random and deterministic parameters.

Most of the lower bounds assume a white observation noise and a stationary signal.

In this contribution, we consider an oversampled (regarding the symbol time interval) signal model after receiver matched filtering, this implies dealing with a coloured reception noise and taking into account the non-stationarity of the digital signal power (cyclostationarity when transmitting a random sequence), as done in [3]. Although this scenario is standard in satellite radio-localization based on a BOC time-limited shaping pulse modulation, there is no theoretical study concerning the performance of the dynamical oversampled phase and frequency offset estimation in this context (to the best of our knowledge). We derive a closed-form expression of the on-line Hybrid Cramér-Rao Bound (HCRB) for the dynamical phase and frequency offset estimation in a Data Aided (DA) scenario, assuming a Brownian phase evolution with a linear drift. The study allows to measure the potential gain for phase and frequency offset estimation provided by the use of the fractionally-spaced processing after matched filtering, instead of the symbol time-spaced signal. In [4] we propose an algorithm that approach this lower bound.

This paper is organized as follows. Section II sets the signal model. Section III recalls the HCRB expressions and derives the HCRB for this estimation problem. Finally, in Section IV, the numerical results are presented and interpreted. The conclusion is given in Section V.

Notations: italic indicates a scalar quantity, as in $a$; boldface indicates a vector quantity, as in a and capital boldface indicates a matrix quantity as in $\mathbf{A}$. The $(k, l)^{t h}$ entry of a matrix $\mathbf{A}$ is denoted $[\mathbf{A}]_{k, l}$. The matrix transpose and selfadjoint operators are denoted by the superscripts $T$ and $H$ respectively as in $\mathbf{A}^{T}$ and $\mathbf{A}^{H} \cdot \Re(\cdot), \Im(\cdot)$ and $(\cdot)^{*}$ are the real part, the imaginary part and conjugate of a complex number or matrix, respectively. $\mathrm{E}_{x}$ denotes the expectation over $x . \nabla_{\boldsymbol{\theta}}$ and $\Delta_{\psi}^{\boldsymbol{\theta}}$,represent the first and second-order partial derivatives operator i.e., $\nabla_{\boldsymbol{\theta}}=\left[\frac{\partial}{\partial \theta_{1}} \cdots \frac{\partial}{\partial \theta_{K}}\right]^{T}$ and $\Delta_{\boldsymbol{\psi}}^{\boldsymbol{\theta}}=\nabla_{\boldsymbol{\psi}} \nabla_{\boldsymbol{\theta}}^{T}$.

\section{Signal MODEL}

We propose the signal model for the transmission of a known complex-valued sequence $\left\{a_{m}\right\}_{m \in \mathbb{Z}}$ over an Additive White Gaussian Noise (AWGN) channel affected by a carrier phase offset $\theta(t)$.

\section{A. Oversampled Signal Model}

The received complex baseband signal after matched filtering is

$$
y(t)=\left[\left\{T \sum_{m} a_{m} \Pi(t-m T)\right\} e^{i \theta(t)}+n(t)\right] * \Pi^{*}(-t)
$$


where $T, \Pi(t)$ and $n(t)$ stands for the symbol period, shaping pulse and circular gaussian noise with a known bilateral power spectral density (psd) $N_{0}$.

We define the filtered coloured noise

$$
b(t)=[n(t)] * \Pi^{*}(-t)
$$

and the shaping function $\tilde{g}_{m}(t)$ as

$$
\tilde{g}_{m}(t)=T \int_{-\infty}^{+\infty} \Pi^{*}(-\alpha) e^{i \theta(t-\alpha+m T)} \Pi(t-\alpha) d \alpha
$$

Then the received signal can be written as

$$
y(t)=\sum_{m} a_{m} \tilde{g}_{m}(t-m T)+b(t)
$$

Hereafter we suppose a shaping pulse with support in $[0, T]$ and a slow varying phase evolution during a period $T$. In this case we can approximate $\tilde{g}_{m}(t)$ by

$$
\tilde{g}_{m}(t) \approx g(t) e^{i \theta\left(t+\left(m+\frac{1}{2}\right) T\right)}
$$

where

$$
g(t)=T \int_{-T}^{0} \Pi^{*}(-\alpha) \Pi(t-\alpha) d \alpha
$$

If the received signal is fractionally-spaced at $t_{k}=k \frac{T}{S}+\tau$, where $S$ is an integer oversampling factor and $\tau$ a known offset from the optimum sampling instants (we suppose $0 \leq \tau<\frac{T}{S}$ ), we have that

$$
y\left(k \frac{T}{S}+\tau\right)=\sum_{m} a_{m} \tilde{g}_{m}\left(k \frac{T}{S}+\tau-m T\right)+b\left(k \frac{T}{S}+\tau\right)
$$

and from (eq. 5) we have that

$$
y\left(k \frac{T}{S}+\tau\right)=e^{i \theta\left(k \frac{T}{S}+\tau+\frac{T}{2}\right)} A_{k}+b\left(k \frac{T}{S}+\tau\right)
$$

where

$$
A_{k}=\sum_{m} a_{m} g\left(k \frac{T}{S}+\tau-m T\right)
$$

We can finally write the received oversampled signal as

$$
y_{k}=A_{k} e^{i \theta_{k}}+b_{k}
$$

where $k$ refers to $t_{k}$ instants. Note that the noise $b_{k}$ is coloured with variance $\sigma_{n}^{2}$, where $\sigma_{n}^{2}=N_{0} \times \frac{g(0)}{T}$ is the variance of the AWGN $n(t)$ measured in the noise equivalent bandwidth of the receiver filter $\Pi^{*}(-t)$.

We can define the symbol index $p=\left\lfloor\frac{k}{S}\right\rfloor$, or equivalently, $k=p S+s$ with $s$ the sub-symbol index (i.e. the position inside the symbol interval). We note that $s=0, \cdots, S-1$.

We note that $\left\{A_{k}\right\}_{k \in \mathbb{Z}}$ is a non-stationary power sequence for $S>1$, even if $\left\{a_{m}\right\}_{m \in \mathbb{Z}}$ is a stationary power symbol sequence $\left(a_{m}^{2}=1\right)$.

\section{B. Phase-offset Evolution Model}

In practice we have a frequency shift between transmitter's ocillator and receiver's oscillator, so the phase distortion is linear. We also must consider jitters introduced by oscillators imperfections. To take it into account we suppose a Brownian phase offset evolution with a linear drift [5]:

$$
\theta_{k}=\theta_{k-1}+\delta+w_{k} \quad k \geq 2
$$

where $w_{k}$ is an i.i.d. zero-mean Gaussian noise sequences with known variance $\frac{\sigma_{w}}{S}$, where $\sigma_{w}^{2}$ stands for the variance of the phase increment in one symbol interval, and $\delta$ is the unknown constant drift. We note that the variance of the Gaussian noise is directly related with the rapidity of evolution of the parameter. We note $\boldsymbol{\theta}=\left[\theta_{1} \cdots \theta_{N}\right]^{T}$ with a covariance matrix $\Sigma$.

\section{HYBRID CRAMÉR-RAO BOUND}

When dealing with an estimation problem we aim to know the ultimate accuracy that can be achieved by the estimator. The Cramér-Rao Bounds (CRB) provide a lower bound on the Mean Square Error (MSE) achievable by any unbiased estimator. Depending on the nature of the parameters to be estimated we use different bounds of the CRB family. If the vector of parameters is assumed to be deterministic we use the standard CRB and if the vector of parameters is random and an a priori information is available we use the so-called Bayesian CRB [6]. When dealing with both random and deterministic parameters an Hybrid CRB (HCRB) is used [7]. The CRB suited to our problem is the HCRB as we want to estimate the phase offset evolution vector $\boldsymbol{\theta}$ which is a random vector with an a priori probability density function (pdf) $p(\boldsymbol{\theta})$ and the linear drift which is a deterministic parameter.

In the on-line synchronization mode, at time $k$ the receiver updates the observation vector $\mathbf{y}=\left[y_{1} \cdots y_{k-1}\right]^{T}$ including the new observation $y_{k}$ to obtain the updated vector $\mathbf{y}=$ $\left[y_{1} \cdots y_{k}\right]^{T}$ in order to estimate $\theta_{k}$. In this section we recall the expression of the Hybrid CRB and we present the closedform expression of the HCRB for an oversampled dynamical phase and frequency offset estimation problem in a Data Aided scenario.

\section{A. HCRB: background}

We have a set of measurements $\mathbf{y}$ and we want to estimate a $N$-dimensional vector of parameters $\boldsymbol{\mu}=\left(\boldsymbol{\mu}_{r}^{T} \boldsymbol{\mu}_{d}^{T}\right)^{T}$. We consider the case where the random $\left(\boldsymbol{\mu}_{r}\right)$ and the deterministic $\left(\boldsymbol{\mu}_{d}\right)$ part of the vector of parameters can be statistically dependent. We note $\boldsymbol{\mu}_{d}^{*}$ the true value of $\boldsymbol{\mu}_{d}$. The joint probability density of the pair $(\mathbf{y}, \boldsymbol{\mu})$ is $p_{\mathbf{y}, \boldsymbol{\mu}}(\mathbf{y}, \boldsymbol{\mu})$ and the a priori pdf of the random part of $\boldsymbol{\mu}$ is $p\left(\boldsymbol{\mu}_{r} \mid \boldsymbol{\mu}_{d}^{*}\right) \neq p\left(\boldsymbol{\mu}_{r}\right)$. If $\hat{\boldsymbol{\mu}}(\mathbf{y})$ is our estimate of $\boldsymbol{\mu}$, the HCRB satisfies the following inequality on the MSE:

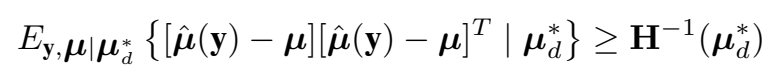

where $\mathbf{H}\left(\boldsymbol{\mu}_{d}^{*}\right)$ is the so-called Hybrid Information Matrix (HIM) defined as [7]

$$
\mathbf{H}\left(\boldsymbol{\mu}_{d}^{*}\right)=E_{\mathbf{y}, \boldsymbol{\mu}_{r} \mid \boldsymbol{\mu}_{d}^{*}}\left[-\Delta_{\boldsymbol{\mu}}^{\boldsymbol{\mu}} \log p\left(\mathbf{y}, \boldsymbol{\mu}_{r} \mid \boldsymbol{\mu}_{d}\right) \mid \boldsymbol{\mu}_{d}^{*}\right]
$$


Expanding the log-likelihood the HIM can be rewritten as

$$
\begin{aligned}
& \mathbf{H}\left(\boldsymbol{\mu}_{d}^{*}\right)=E_{\boldsymbol{\mu}_{r} \mid \boldsymbol{\mu}_{d}^{*}}\left[\mathbf{F}\left(\boldsymbol{\mu}_{r}, \boldsymbol{\mu}_{d}^{*}\right)\right] \\
& +E_{\boldsymbol{\mu}_{r} \mid \boldsymbol{\mu}_{d}^{*}}\left[-\Delta_{\boldsymbol{\mu}}^{\boldsymbol{\mu}} \log p\left(\boldsymbol{\mu}_{r} \mid \boldsymbol{\mu}_{d}\right) \mid \boldsymbol{\mu}_{d}^{*}\right]
\end{aligned}
$$

where $\mathbf{F}\left(\boldsymbol{\mu}_{r}, \boldsymbol{\mu}_{d}^{*}\right)$ is the Fisher Information Matrix (FIM) defined as

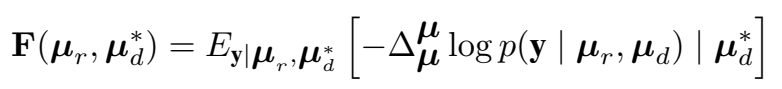

We can see that $\mathbf{H}\left(\boldsymbol{\mu}_{d}^{*}\right)=\mathbf{H}^{D}\left(\boldsymbol{\mu}_{d}^{*}\right)+\mathbf{H}^{P}\left(\boldsymbol{\mu}_{d}^{*}\right)$, where the first term represents the average information about $\boldsymbol{\mu}$ brought by the observations $\mathbf{y}$ and the second term represents the information available from the prior knowledge on $\boldsymbol{\mu}$, i.e., $p\left(\boldsymbol{\mu}_{r} \mid \boldsymbol{\mu}_{d}\right)$.

The $N \times N$ HCRB matrix can be written as

$$
\text { HCRB }=\left\{\mathbf{H}\left(\boldsymbol{\mu}_{d}^{*}\right)\right\}^{-1}=\left\{\mathbf{H}^{D}\left(\boldsymbol{\mu}_{d}^{*}\right)+\mathbf{H}^{P}\left(\boldsymbol{\mu}_{d}^{*}\right)\right\}^{-1}
$$

where the $k^{t h}$ element of the diagonal, $[\mathbf{H C R B}]_{k, k}$ represents the lower bound on the estimation of $[\boldsymbol{\mu}]_{k}$ from the observations block $\mathbf{y}=\left[y_{1} \cdots y_{N}\right]$.

\section{B. HCRB: Application to Dynamical Phase Offset and Linear Drift Estimation}

In this paragraph, a closed-form expression for the HCRB for an on-line fractionally-spaced phase-offset and linear drift estimation problem is presented. On the following we drop the dependence of the different matrices on $\boldsymbol{\mu}_{d}^{*}=\delta^{*}$ for easier notation.

We use the model presented in Section II (eqs.11,10):

$$
\begin{aligned}
& \theta_{k}=\theta_{k-1}+\delta+w_{k} \\
& y_{k}=A_{k} \exp \left(i \theta_{k}\right)+b_{k}
\end{aligned}
$$

where, as stated before, $b_{k}$ is a non-white noise with covariance matrix $\boldsymbol{\Gamma}$. The index $k$ refers to $t_{k}$ instants and $A_{k}$ are the coefficients specified in eq.(9).

Comparing this state-space model to the general model presented on the last paragraph, and supposing that we have $N$ available measurements, we identify $\boldsymbol{\mu}_{r}=\boldsymbol{\theta}=\left[\theta_{1} \cdots \theta_{N}\right]^{T}$ and $\boldsymbol{\mu}_{d}=\delta$. From this the HIM can be rewritten into a $(N+1) \times(N+1)$ block matrix as [7]

$$
\mathbf{H}=\left(\begin{array}{cc}
\mathbf{H}_{11} & \mathbf{h}_{12} \\
\mathbf{h}_{21} & H_{22}
\end{array}\right)
$$

where

$$
\begin{aligned}
\mathbf{H}_{11}= & E_{\mathbf{y}, \boldsymbol{\theta} \mid \delta^{*}}\left[-\Delta_{\boldsymbol{\theta}}^{\boldsymbol{\theta}} \log p(\mathbf{y} \mid \boldsymbol{\theta}, \delta) \mid \delta^{*}\right] \\
& +E_{\boldsymbol{\theta} \mid \delta^{*}}\left[-\Delta_{\boldsymbol{\theta}}^{\boldsymbol{\theta}} \log p\left(\boldsymbol{\theta} \mid \delta^{*}\right)\right] \\
\mathbf{h}_{12}= & \mathbf{h}_{21}^{T}=E_{\mathbf{y}, \boldsymbol{\theta} \mid \delta^{*}}\left[-\Delta_{\boldsymbol{\theta}}^{\delta} \log p(\mathbf{y} \mid \boldsymbol{\theta}, \delta) \mid \delta^{*}\right] \\
& +E_{\boldsymbol{\theta} \mid \delta^{*}}\left[-\Delta_{\boldsymbol{\theta}}^{\delta} \log p\left(\boldsymbol{\theta} \mid \delta^{*}\right)\right] \\
H_{22}= & E_{\mathbf{y}, \boldsymbol{\theta} \mid \delta^{*}}\left[-\Delta_{\delta}^{\delta} \log p(\mathbf{y} \mid \boldsymbol{\theta}, \delta) \mid \delta^{*}\right] \\
& +E_{\boldsymbol{\theta} \mid \delta^{*}}\left[-\Delta_{\delta}^{\delta} \log p\left(\boldsymbol{\theta} \mid \delta^{*}\right)\right]
\end{aligned}
$$

So to compute the HIM we need the likelihood function and the a priori pdf. From the model we can write the loglikelihood as

$$
\log p\left(\mathbf{y} \mid \boldsymbol{\theta}, \delta^{*}\right)=\log \frac{1}{\pi^{N}|\operatorname{det}(\boldsymbol{\Gamma})|}-[\mathbf{y}-\mathbf{m}]^{H} \boldsymbol{\Gamma}^{-1}[\mathbf{y}-\mathbf{m}]
$$

where $\mathbf{y}$ is the $N$-dimensional received signal array and $\mathbf{m}$ is the mean vector of $\mathbf{y}$, where the $k^{t h}$ component is $[\mathbf{m}]_{k}=$ $A_{k} e^{i \theta_{k}}$. The a priori pdf is

$$
\begin{aligned}
\log p\left(\boldsymbol{\theta} \mid \delta^{*}\right)= & \log p\left(\theta_{1}\right)+(N-1) \log \left(\frac{1}{\sqrt{2 \pi} \sigma_{w}}\right) \\
& -\sum_{k=2}^{N} \frac{\left(\theta_{k}-\theta_{k-1}-\delta^{*}\right)^{2}}{2 \sigma_{w}^{2}}
\end{aligned}
$$

- Expression of $\mathbf{H}_{11}$ : we can write that

$$
\mathbf{H}_{11}=\mathbf{H}_{11}^{D}+\mathbf{H}_{11}^{P}
$$

where

$$
\begin{gathered}
\mathbf{H}_{11}^{D}=E_{\mathbf{y}, \boldsymbol{\theta} \mid \delta^{*}}\left[-\Delta_{\boldsymbol{\theta}}^{\boldsymbol{\theta}} \log p(\mathbf{y} \mid \boldsymbol{\theta}, \delta) \mid \delta^{*}\right] \\
\mathbf{H}_{11}^{P}=E_{\boldsymbol{\theta} \mid \delta^{*}}\left[-\Delta_{\boldsymbol{\theta}}^{\boldsymbol{\theta}} \log p\left(\boldsymbol{\theta} \mid \delta^{*}\right)\right]
\end{gathered}
$$

The first term can be computed from eq.(17). We note $\Lambda(\boldsymbol{\theta})=\log p(\mathbf{y} \mid \boldsymbol{\theta}, \delta)$. The first derivative of $\Lambda(\boldsymbol{\theta})$ with respect to the $l^{\text {th }}$ phase parameter is

$$
\begin{aligned}
\frac{\partial \Lambda(\boldsymbol{\theta})}{\partial \theta_{l}} & =\frac{\partial}{\partial \theta_{l}}\left\{-[\mathbf{y}-\mathbf{m}]^{H} \boldsymbol{\Gamma}^{-1}[\mathbf{y}-\mathbf{m}]\right\} \\
& =\left\{\frac{\partial \mathbf{m}^{H}}{\partial \theta_{l}} \boldsymbol{\Gamma}^{-1}[\mathbf{y}-\mathbf{m}]+[\mathbf{y}-\mathbf{m}]^{H} \boldsymbol{\Gamma}^{-1} \frac{\partial \mathbf{m}}{\partial \theta_{l}}\right\} \\
& =2 \Re\left\{\frac{\partial \mathbf{m}^{H}}{\partial \theta_{l}} \boldsymbol{\Gamma}^{-1}[\mathbf{y}-\mathbf{m}]\right\}
\end{aligned}
$$

if we compute now the derivative with respect to the $k^{t h}$ phase parameter we have that

$$
\frac{\partial^{2} \Lambda(\boldsymbol{\theta})}{\partial \theta_{k} \partial \theta_{l}}=2 \Re\left\{\frac{\partial^{2} \mathbf{m}^{H}}{\partial \theta_{k} \partial \theta_{l}} \boldsymbol{\Gamma}^{-1}[\mathbf{y}-\mathbf{m}]-\frac{\partial \mathbf{m}^{H}}{\partial \theta_{l}} \boldsymbol{\Gamma}^{-1} \frac{\partial \mathbf{m}}{\partial \theta_{k}}\right\}
$$

The $(k, l)^{t h}$ element of the matrix $\mathbf{H}_{11}^{D}$ is

$$
\begin{aligned}
{\left[\mathbf{H}_{11}^{D}\right]_{k, l} } & =E_{\boldsymbol{\theta} \mid \delta^{*}}\left\{E_{\mathbf{y} \mid \boldsymbol{\theta}, \delta^{*}}\left\{-\frac{\partial^{2} \Lambda(\boldsymbol{\theta})}{\partial \theta_{k} \partial \theta_{l}}\right\}\right\} \\
& =E_{\boldsymbol{\theta} \mid \delta^{*}}\left\{2 \Re\left\{\frac{\partial \mathbf{m}^{H}}{\partial \theta_{l}} \boldsymbol{\Gamma}^{-1} \frac{\partial \mathbf{m}}{\partial \theta_{k}}\right\}\right\}
\end{aligned}
$$

We note that

$$
\begin{array}{r}
\frac{\partial \mathbf{m}^{H}}{\partial \theta_{l}}=\left[0, \cdots, 0,-i A_{l}^{*} e^{-i \theta_{l}}, 0, \cdots, 0\right] \\
\frac{\partial \mathbf{m}}{\partial \theta_{k}}=\left[0, \cdots, 0, i A_{k} e^{i \theta_{k}}, 0, \cdots, 0\right]^{T}
\end{array}
$$

with the non-null values on the $l^{t h}$ and $k^{t h}$ position respectively, and so the coefficients can be written as

$$
\begin{aligned}
{\left[\mathbf{H}_{11}^{D}\right]_{k, l} } & =E_{\boldsymbol{\theta} \mid \delta^{*}}\left\{2 \Re\left\{A_{l}^{*} A_{k} \cdot\left[\boldsymbol{\Gamma}^{-1}\right]_{k, l} e^{j\left(\theta_{k}-\theta_{l}\right)}\right\}\right\} \\
& =2 \Re\left\{A_{l}^{*} A_{k} \cdot\left[\boldsymbol{\Gamma}^{-1}\right]_{k, l} E_{\boldsymbol{\theta} \mid \delta^{*}}\left\{e^{j\left(\theta_{k}-\theta_{l}\right)}\right\}\right\}
\end{aligned}
$$


We can write that

$$
E_{\boldsymbol{\theta}}\left\{e^{i\left(\theta_{k}-\theta_{l}\right)}\right\}=E_{\boldsymbol{\theta}}\left\{e^{i\left(\mathbf{u}_{k l}^{T} \boldsymbol{\theta}\right)}\right\}
$$

where $\mathbf{u}_{k l}^{T}=[0, \cdots, 0,(+1), 0, \cdots, 0,(-1), 0, \cdots, 0]$, +1 in the $k^{t h}$ position and -1 in the $l^{t h}$ position of the array, $\phi(\cdot)$ is the characteristic function of a Gaussian random variable $\boldsymbol{\theta}$ :

$$
\begin{aligned}
\phi\left(\mathbf{u}_{k l}\right) & =\exp \left\{-\frac{1}{2} \mathbf{u}_{k l}^{T} \boldsymbol{\Sigma}^{-1} \mathbf{u}_{k l}\right\} \\
& =\exp \left\{-\frac{1}{2}\left(\left[\boldsymbol{\Sigma}^{-1}\right]_{k, k}+\left[\boldsymbol{\Sigma}^{-1}\right]_{l, l}-2\left[\boldsymbol{\Sigma}^{-1}\right]_{k, l}\right)\right\}
\end{aligned}
$$

with $\boldsymbol{\Sigma}$ the covariance matrix of the phase evolution $\boldsymbol{\theta}$. Finally

$$
\left[\mathbf{H}_{11}^{D}\right]_{k, l}=2 \Re\left\{A_{l}^{*} A_{k}\left[\boldsymbol{\Gamma}^{-1}\right]_{k, l} e^{\Psi}\right\}
$$

where

$$
\Psi=\left\{-\frac{1}{2}\left(\left[\boldsymbol{\Sigma}^{-1}\right]_{k, k}+\left[\boldsymbol{\Sigma}^{-1}\right]_{l, l}-2\left[\boldsymbol{\Sigma}^{-1}\right]_{k, l}\right)\right\}_{0}
$$

On the following we compute the second term of eq.(19). From the state evolution eq.(11) and assuming that the initial phase $\theta_{1}$ doesn't depend on $\delta$, we have that

$$
p\left(\boldsymbol{\theta} \mid \delta^{*}\right)=p\left(\theta_{1}\right) \Pi_{k=2}^{N} p\left(\theta_{k} \mid \theta_{k-1}, \delta^{*}\right)
$$

and due to this expansion we can rewrite the expression as

$$
\Delta_{\boldsymbol{\theta}}^{\boldsymbol{\theta}} \ln p\left(\boldsymbol{\theta}, \delta^{*}\right)=\Delta_{\boldsymbol{\theta}}^{\boldsymbol{\theta}} \ln p\left(\theta_{1}\right)+\sum_{k=1}^{N} \Delta_{\boldsymbol{\theta}}^{\boldsymbol{\theta}} \ln p\left(\theta_{k} \mid \theta_{k-1}, \delta^{*}\right)
$$

The first term in (eq.29) is a matrix with only one nonzero element, namely, the entry $(1,1)$ which is equal to

$$
\left[\Delta_{\boldsymbol{\theta}}^{\boldsymbol{\theta}} \ln p\left(\theta_{1}\right)\right]_{1,1}=\frac{\partial^{2} \ln p\left(\theta_{1}\right)}{\partial \theta_{1}^{2}}
$$

The other terms are matrices with only four non-zero elements, namely, the entries $(k-1, k-1),(k-1, k)$, $(k, k-1)$ and $(k, k)$. Due to the Gaussian nature of the noise, one finds

$$
\begin{aligned}
{\left[\Delta_{\boldsymbol{\theta}}^{\boldsymbol{\theta}} \ln p\left(\theta_{k} \mid \theta_{k-1}, \boldsymbol{\delta}^{*}\right)\right]_{k, k} } & =\frac{-s}{\sigma_{w}^{2}} \\
{\left[\Delta_{\boldsymbol{\theta}}^{\boldsymbol{\theta}} \ln p\left(\theta_{k} \mid \theta_{k-1}, \boldsymbol{\delta}^{*}\right)\right]_{k, k-1} } & =\frac{s}{\sigma_{w}^{2}}
\end{aligned}
$$

The values for $(k-1, k-1)$ and $(k-1, k)$ are, respectively, the same that for $(k, k)$ and $(k-1, k)$. Assuming that $E_{\theta_{1}}\left[\Delta_{\boldsymbol{\theta}}^{\boldsymbol{\theta}} \ln p\left(\theta_{1}\right)\right]=0$ that corresponds to the case of non-informative prior about $\theta_{1}$ (see [2]), we obtain that

$$
\begin{gathered}
\mathbf{H}_{11}^{P}=E_{\boldsymbol{\theta} \mid \delta^{*}}\left[-\Delta_{\boldsymbol{\theta}}^{\boldsymbol{\theta}} \log p\left(\boldsymbol{\theta} \mid \delta^{*}\right)\right] \\
=\frac{1}{\sigma_{w}^{2} / s}\left(\begin{array}{ccccc}
1 & -1 & 0 & \cdots & 0 \\
-1 & 2 & -1 & \ddots & \vdots \\
0 & \ddots & \ddots & \ddots & 0 \\
\vdots & & -1 & 2 & -1 \\
0 & \ldots & 0 & -1 & 1
\end{array}\right)
\end{gathered}
$$

- Expression of $\mathbf{h}_{12}$ : the log-likelihood (eq.17) doesn't depend on $\delta$ so the first term of $\mathbf{h}_{12}$ is null, so

$$
\mathbf{h}_{12}=E_{\boldsymbol{\theta} \mid \delta^{*}}\left[-\Delta_{\boldsymbol{\theta}}^{\delta} \log p\left(\boldsymbol{\theta} \mid \delta^{*}\right)\right]
$$

From the state model we have that

$$
\mathbf{h}_{12}=\left[\frac{S}{\sigma_{w}^{2}} \mathbf{0}_{1 \times N-2}-\frac{S}{\sigma_{w}^{2}}\right]^{T}
$$

- Expression of $H_{22}$ : as the log-likelihood doesn't depend on $\delta$ and using eq.(18) we have that

$$
\begin{aligned}
H_{22} & =E_{\boldsymbol{\theta} \mid \delta^{*}}\left[-\Delta_{\delta}^{\delta} \log p\left(\boldsymbol{\theta} \mid \delta^{*}\right)\right] \\
& =E_{\boldsymbol{\theta} \mid \delta^{*}}\left[-\frac{\partial^{2}}{\partial \delta^{2}} \log p\left(\boldsymbol{\theta} \mid \delta^{*}\right)\right] \\
& =\frac{S(N-1)}{\sigma_{w}^{2}}
\end{aligned}
$$

Remarks: we as analyse the estimation problem in a DA scenario the bound depends on the transmitted sequence $\mathbf{a}$. We note that, contrary to [3] where the proposed bound was the minimum over a set of sequences, the HCRB is computed in this paper for a specific known sequence.

However we note that for $S=1, s=0$ (symbol reference points) and $\tau=0$, we have that the bound is independent of the transmitted sequence a since $\left|A_{k}\right|=1 \forall k$. In the other cases the bound depends on the sequence, the oversampling factor $S$ and on the position $s$ inside the symbol interval of the current transmitted symbol (index $M$ ):

$$
\begin{aligned}
H C R B_{\theta}(\mathbf{a}, S, s) & =\left[\mathbf{H}^{-1}(\mathbf{a})\right]_{N, N} \\
H C R B_{\delta}(\mathbf{a}, S, s) & =\left[\mathbf{H}^{-1}(\mathbf{a})\right]_{N+1, N+1}
\end{aligned}
$$

with $N=(M-1) * S+1+s$.

\section{Discussion}

In this section we show the behaviour of the HCRB by considering different scenarios. We assume the transmission over an AWGN channel of a known M-sequence of length 511 bits, generated using a Linear Feedback Shift Register (LFSR) with characteristic polynomial $[1021]_{8}$ (octal representation). We consider three oversampling factors $(S=1,2$ and 4$)$ and a BOC shaping pulse (see figure 1). BOC shaping pulse is used in Galileo positioning system.

In the figures, we plot the Mean Square Error (MSE) obtained by Monte Carlo simulation versus the Signal to Noise Ratio (SNR). The SNR corresponds to the Carrier to Noise Ratio $\left(\frac{C}{N}\right)$ at the input of the receiver. In our case, as shaping pulse and symbols $a_{k}$ are normalised (i.e $\sigma_{a}^{2}=1 ; g(0)=1$ ) 

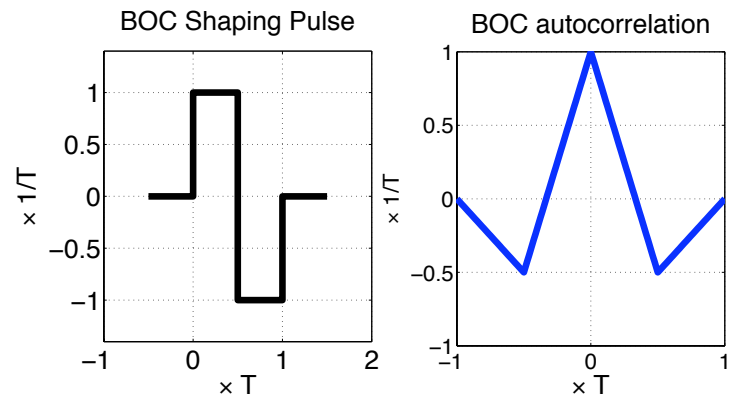

Fig. 1. BOC shaping function $\Pi(t)$ and its autocorrelation $g(t)$

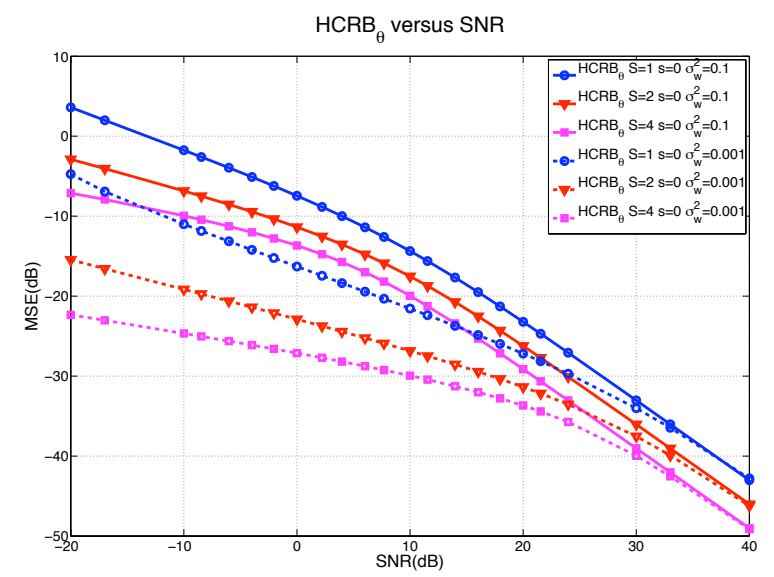

Fig. 2. HCRB versus the SNR for three different oversampling factors $S=$ 1,2 and 4 . We consider two phase noise variances $\sigma_{w}^{2}=0.001 \mathrm{rad}^{2}$ and $\sigma_{w}^{2}=0.1 \mathrm{rad}^{2}$.

this ratio is simply $\frac{C}{N}=\frac{1}{\sigma_{n}^{2}}$. For the MSE we consider two cases:

1) We compute $B C R B(\mathbf{a}, S, 0)$ for the $T$-spaced symbol reference point estimation for $S=1,2,4$.

2) We compute the MSE for the mid-point estimation ( $s=$ $S / 2$ ). This scenario is also interesting because the intermediate points can be useful when using fractionallyspaced algorithms (i.e. half symbol spaced equalizer or Gardner's timing recovery algorithm).

\section{A. T-spaced symbol reference point estimation MSE}

Figure 2 superimposes versus the SNR, the on-line HCRB (see eq.(34)) for two different phase variance values. We consider a slow varying phase with variance $\sigma_{w}^{2}=0.001 \mathrm{rad}^{2}$ and a phase with a fast evolution, $\sigma_{w}^{2}=0.1 \mathrm{rad}^{2}$. In both cases there's no offset from the optimal sampling instants, $\tau=0$.

One can see that the gain increases with the oversampling factor $S$ and the interest of oversampling becomes clear at low SNR. The gain due to oversampling decreases as the SNR increases. As expected, we note that the performance are looser when increasing the phase noise variance.

In figure 3 we analyse the HCRB behaviour versus phase noise variance for a slow SNR value $(0 \mathrm{~dB})$. Here we can still measure the potential gain given by the oversampling. The

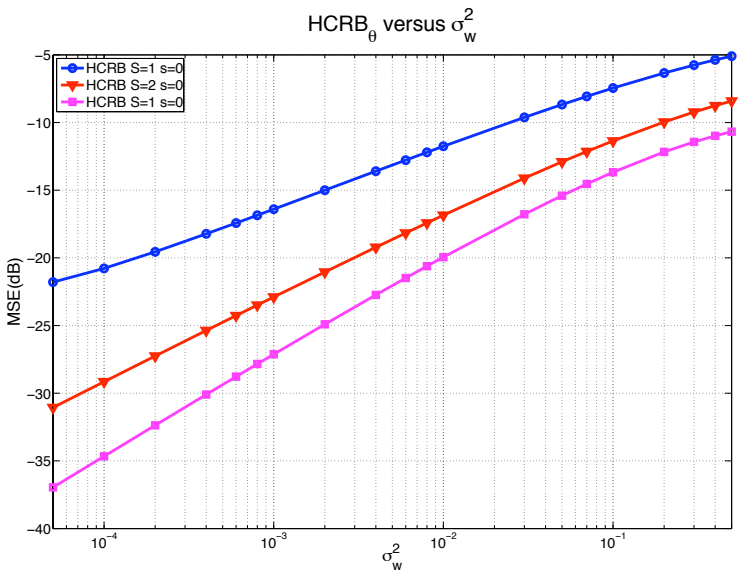

Fig. 3. HCRB versus the phase noise variance for three different oversampling factors $S=1,2$ and $4, \mathrm{SNR}=0 \mathrm{~dB}$.

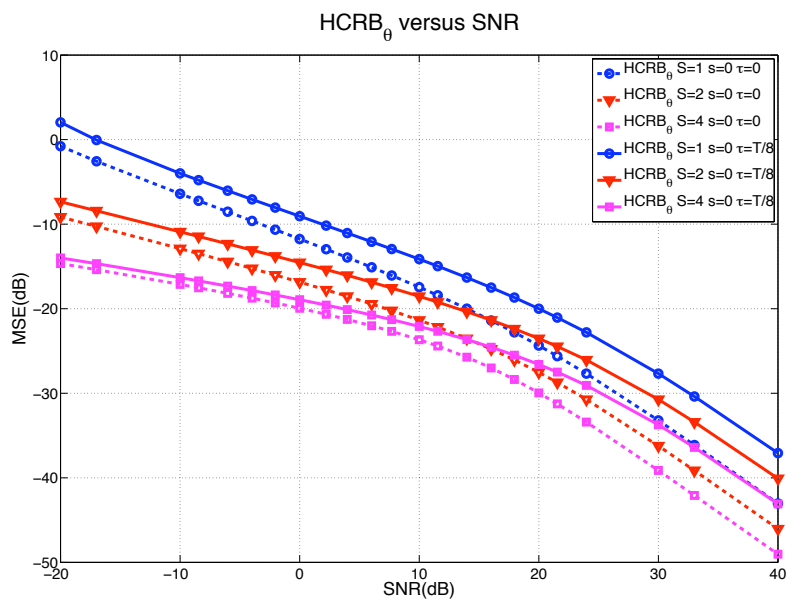

Fig. 4. HCRB versus the SNR for three different oversampling factors $S=$ 1,2 and 4 . We consider a phase noise variance $\sigma_{w}^{2}=0.01 \operatorname{rad}^{2}$ and in presence of a non nul offset for the sampling instants.

gain obtained with the oversampling is greater at weak $\sigma_{w}^{2}$.

Figure 4 superimposes versus the SNR, the on-line HCRB, $H C R B_{\theta}(\mathbf{a}, S, 0)$, for a moderate varying phase evolution, $\sigma_{w}^{2}=0.01$, and a non-null offset $\tau=\frac{T}{8}$. For $\tau \neq 0$, the bound are looser, performances decrease when increasing $\tau$. The gain between different oversampling factors is greater at high SNR when having a non-null offset.

Figure 5 superimposes versus the SNR, the on-line HCRB for the drift estimation, $\operatorname{HCRB}_{\delta}(\mathbf{a}, S, 0)$, for two different block sizes $N=200$ and $N=511$. We consider a phase noise variance $\sigma_{w}^{2}=0.01$. The performances depend on the estimation block size $N$ because the parameter to be estimated has a constant value. The performances increase when increasing $S$ but in all the cases we have a very good estimation, as shown by weak MSE values.

\section{B. T-spaced symbol mid-points estimation MSE}

We now take the $S=4$ case as the reference. For $S=$ 1 , there is only 1 estimate per symbol, so, to compare with 


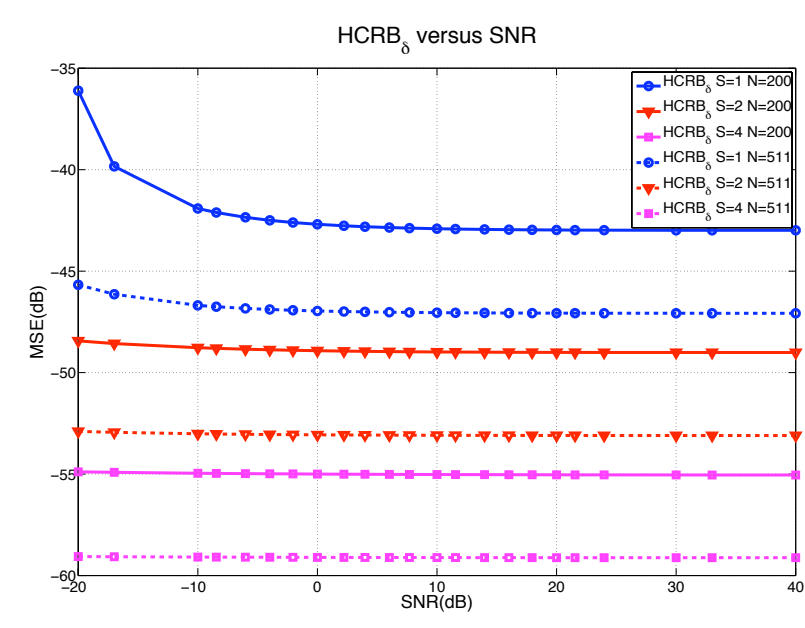

Fig. 5. $H C R B_{\delta}$ versus the SNR for three different oversampling factors $S=1,2$ and 4 . We consider a phase noise variance $\sigma_{w}^{2}=0.01 \mathrm{rad}^{2}$.

$S=2,4$, missing mid-points are generated by blocking the estiamted value $(s=0)$ over the symbol period.

The HCRB for the blocked case $S=1$ can be easily obtained as

$$
H C R B_{\theta-b}(\mathbf{a}, 1,1 / 2)=\operatorname{HCRB}_{\theta}(\mathbf{a}, 1,0)+\sigma_{w}^{2} / 2 .
$$

For $S>1$, the bound strongly depends on the last observed value $A_{N}$ because of the non-stationary power. In this case we rename the bound as $B C R B\left(A_{N}, S, s\right)$. For $\tau=0,\left|A_{N}\right|$ can be equal to 0 or 1 , so there are two possible bounds. We can define an average bound as follows:

$$
\begin{aligned}
\operatorname{HCRB}_{\theta}(S, s)= & p_{0} \cdot H C R B_{\theta}\left(\left|A_{N}\right|=0, S, s\right) \\
& +\left(1-p_{0}\right) \cdot H C R B_{\theta}\left(\left|A_{N}\right|=1, S, s\right)
\end{aligned}
$$

where $p_{0}$ is the proportion of $A_{k}=0$.

1) Results: For the mid-points case, figure 6 superimposes, versus the SNR, the on-line $\mathrm{HCRB}\left(H C R B_{\theta-b}(1,1 / 2)\right.$, $\left.H C R B_{\theta}(2,1), H C R B_{\theta}(4,2)\right)$ for a phase noise variance $\sigma_{w}^{2}=0.01 \mathrm{rad}^{2}$. We also plot as a reference the symbol reference point case.

The gain increases with the oversampling factor $S$, the interest of oversampling, as in the T-spaced reference points case, is clear at low SNR. At high SNR $M S E \rightarrow \sigma_{w}^{2} / 2 S$, this is due to the blocking process for $S=1$ and to the nonstationary power sequence $A_{k}$ for $S=2$ and 4 . We can also see this saturation from eq.(36) which for $p_{0}=0.5$ at high SNR becomes $B C R B(S, s) \sim \frac{1}{2} B C R B(0, S, s)$.

\section{CONClusion}

In the context of satellite positioning systems, like GPS and GALILEO, time limited shaping pulse are used and the Nyquist-Shannon sampling theorem does not apply. These special conditions let us hope a significant receiver synchronization performance improvement when the received signal is oversampled.

In this contribution, we study the gain due to oversampling of the received signal for the problem of dynamical carrier

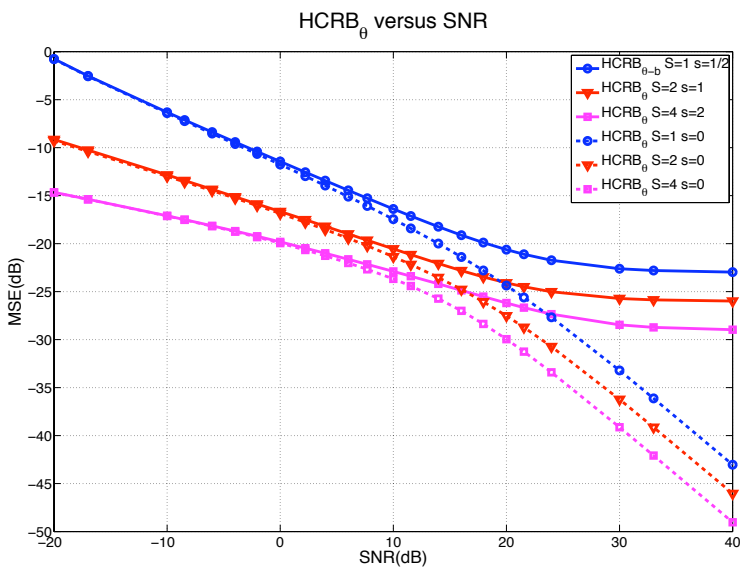

Fig. 6. HCRB versus the SNR for the mid points, three different oversampling factors $S=1,2$ and 4 , and a phase-noise variance $\sigma_{w}^{2}=0.01 \mathrm{rad}^{2}$.

phase and frequency offset tracking. Assuming the data are known at the receiver, we derive the Hybrid Cramér-Rao Bound for carrier phase and frequency offset estimation in such an oversampled scenario.

We present numerical results that show the potential improvements of using a fractionally-spaced method. The estimation MSE decreases as the oversampling factor $S$ increases and the interest of oversampling is more important at low SNR.

\section{ACKNOWLEDGEMENT}

This work was supported in part by the French ANR (Agence Nationale de la Recherche), LURGA project, and the COST Action IC0803 "RF/Microwave Communication Subsystems for Emerging Wireless Technologies (RFCSET).

\section{REFERENCES}

[1] H. L. Van Trees, Detection, Estimation and Modulation Theory. New York: Wiley, 1968, vol.1.

[2] S. M. Kay, Fundamentals of statistical signal processing: estimation theory. Upper Saddle River, NJ, USA: Prentice Hall, Inc., 1993.

[3] J.Vilà Valls, J. M. Brossier and L. Ros, " On-line bayesian CramérRao bound for oversampled dynamical phase offset estimation", IEEE ISCCSP 2008, Malta, 12-14 March 2008.

[4] J.Vilà Valls, J. M. Brossier and L. Ros, " Extended Kalman Filter for oversampled carrier synchronization with large excess bandwidth in digital communications", submitted to IEEE Globecom 2009, Hawaii, 30 Nov-4 Dec 2009.

[5] P. O. Amblard, J. M. Brossier and E. Moisan, " Phase tracking: what do we gain from optimality?Particle filtering versus phase-locked loops", Elsevier Signal Processing, vol. 83, pp. 151-167, Oct. 2003.

[6] S. Bay, C. Herzet, J. M. Brossier, J. P. Barbot, and B. Geller, “ Analytic and Asymptotic Analysis of Bayesian Cramér-Rao Bound for Dynamical Phase Offset Estimation", IEEE Trans. Signal Processing, vol. 56, pp. 61-70, Jan. 2008.

[7] S. Bay, B. Geller, A. Renaux, J. P. Barbot, and J. M. Brossier, "On the Hybrid Cramér Rao Bound and Its Application to Dynamical Phase Estimation”, IEEE Signal Processing Letters, vol. 15, pp. 453-456, 2008.

[8] P. Tichavský, C. H. Muravchik, A. Nehorai, " Posterior Cramér-Rao bounds for discrete-time nonlinear filtering”, IEEE Trans. Signal Processing, vol. 46, pp. 1386-1396, May 1998.

[9] B. M. F. Rice, B. Cowley and M. Rice, " Cramér-Rao Lower Bounds for QAM Phase and Frequency Estimation”, IEEE Trans. Commun., vol. 49, pp. 1582-1591, Sept. 2001

[10] W. G. Cowley, "Phase and frequency estimation for PSK packets: bounds and algorithms", IEEE Trans. Commun., vol. 44, pp. 26-28, Jan. 1996 